\title{
Multiscale analysis of shear failure of thick-walled hollow cylinder in dry sand
}

\author{
N. GUO*, J. ZHAO* and W. C. SUN†
}

\begin{abstract}
A novel hierarchical multiscale model has been applied to simulate the thick-walled hollow cylinder tests in dry sand and to investigate the corresponding shear failures. The combined finite-element method and discrete-element method (FEM/DEM) model employs the FEM as a vehicle to advance the solution for a macroscopic non-linear boundary value problem incrementally. It is, meanwhile, free of conventional macroscopic phenomenological constitutive law, which is replaced by discrete-element simulations conducted with representative volume elements (RVEs) associated with the Gauss quadrature points of the FEM mesh. Numerical simulations proposed by the authors indicate that this multiscale approach is capable of replicating the evolution of cavity pressure during cavity expansion before and after the onset of strain localisation - in qualitative agreement with laboratory tests. In particular, the curvilinear shear bands observed from experiments have been reproduced numerically. The information provided by the mesoscale DEM and the macroscale FEM reveals a close linkage between significant particle rotations taking place inside the dilative shear bands and the highly anisotropic microstructural attributes of the associated RVEs.
\end{abstract}

KEYWORDS: anisotropy; numerical modelling; strain localisation

ICE Publishing: all rights reserved

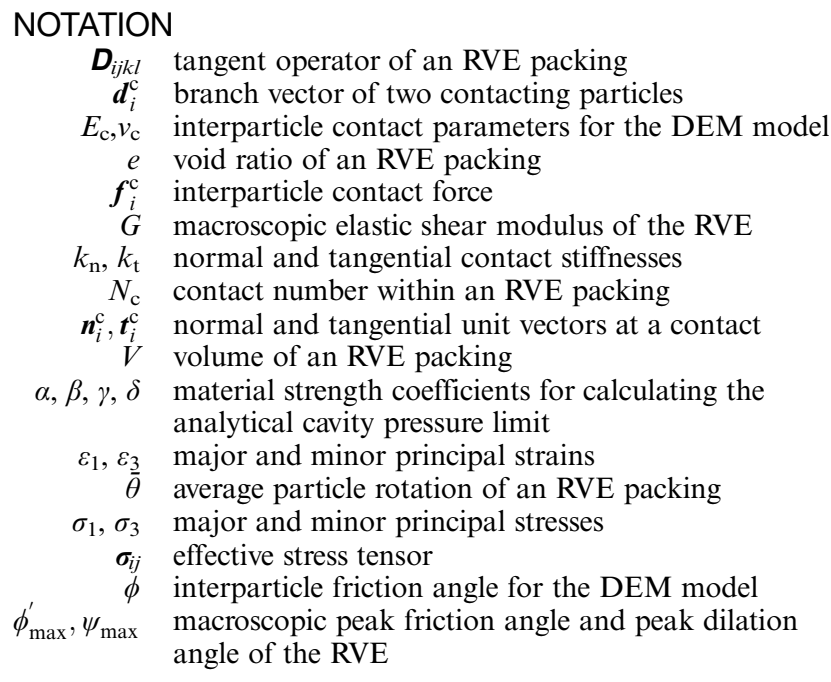

\section{INTRODUCTION}

Cavity expansion in thick-walled hollow cylinder (TWHC) tests is of great importance for practicing geotechnical engineers and researchers (Carter et al., 1986; Mántaras \& Schnaid, 2002). In engineering practice, the mechanical response of soil during cavity expansion dictates the resultant CPT number obtained from the cone penetration test and the estimated shear strength of the soil (Salgado

Manuscript received 30 September 2015; first decision 1 December 2015; accepted 5 December 2015.

Published online at www.geotechniqueletters.com on 11 January 2016.

*Department of Civil and Environmental Engineering, The Hong Kong University of Science and Technology, Kowloon, Hong Kong.

$\dagger$ Department of Civil Engineering and Engineering Mechanics, The Fu Foundation School of Engineering and Applied Science, Columbia University, New York, NY, USA. et al., 1997). Cavity expansion may also occur during the pile-driving process in foundation engineering. It is also an important physical process that affects the stability of boreholes (Yu, 2000).

Cavity expansion replicated in TWHC tests also plays an important role for the theoretical study of soil behaviour. It may provide a complementary perspective to the conventional/true triaxial tests for a better understanding of soil behaviours subjected to complex loading paths. In particular, the intriguing failure patterns in TWHC tests have captured the attention of numerical modellers. In the literature, the bifurcation theory has been applied to predict the shear failure of TWHC based on various phenomenological constitutive models (Vardoulakis \& Papanastasiou, 1988; Papanastasiou \& Vardoulakis, 1989; Anand \& Gu, 2000; Sun, 2013). Notwithstanding their success, these models require a large number of internal variables to capture the inherent anisotropy and evolution of fabric (Zervos et al., 2001; Crook et al., 2003; Sun, 2013, 2015). To overcome these pitfalls, a novel multiscale approach (Kaneko et al., 2003; Miehe \& Dettmar, 2004; Andrade et al., 2011; Guo, 2014; Guo \& Zhao, 2014, 2015; Nguyen et al., 2014; Desrues et al., 2015; Liu et al., 2015b; Zhao \& Guo, 2015a, 2015b) is adopted in this study. The adopted approach employs the finite-element method (FEM) to simulate the macroscopic boundary value problem. Meanwhile, it replaces the phenomenological constitutive law needed in conventional FEM with discrete-element method (DEM) computations at the Gauss quadrature points of the FEM mesh. Each Gauss point is associated with a granular assembly in the size of the representative volume element (RVE). At each loading step, the macroscopic deformation of the granular assemblies is prescribed and the macroscopic Cauchy stress updates are inferred from the deformed RVEs for the global FEM computation.

This multiscale approach provides several advantages. First, the path-dependent behaviours obtained from the simulations are directly related to the grain rearrangements that lead to the formation and collapse/buckling of force 
chains and branch vectors (Tordesillas, 2007; O'Sullivan et al., 2013; Sun et al., 2013a). Hence, there is no need to introduce an excess amount of internal variables to curve-fit the phenomenological responses for specific stress path during cavity expansion (Liu et al., 2015a). Furthermore, the coupling of continuum-scale FEM and grain-scale DEM offers a natural pathway to bridge grain-scale mechanics to field applications. Due to the multiscale coupling, the evolution of microstructural attributes under macroscopic external loading can be examined conveniently. These salient features are exploited to replicate numerically the complex failure patterns commonly observed in the TWHC tests in this study.

\section{APPROACH AND FORMULATION}

In each macroscopic loading step, DEM simulations are performed at the Gauss quadrature points to provide macroscopic constitutive updates. The macroscopic FEM model is solved implicitly by way of the Newton-Raphson iterative scheme. In each iteration, the deformation at each Gauss point is first interpolated by way of the macroscopic shape function and this is subsequently applied as the local boundary condition for each RVE. After the DEM computation, the updated stress tensor $\sigma_{i j}$ and the tangent operator $\boldsymbol{D}_{i j k l}$ can be homogenised using the following equations

$$
\begin{aligned}
& \boldsymbol{\sigma}_{i j}=\frac{1}{V} \sum_{N_{\mathrm{c}}} \boldsymbol{d}_{i}^{\mathrm{c}} \boldsymbol{f}_{j}^{\mathrm{c}} \\
& \boldsymbol{D}_{i j k l}=\frac{1}{V} \sum_{N_{\mathrm{c}}}\left(k_{\mathrm{n}} \boldsymbol{n}_{i}^{\mathrm{c}} \boldsymbol{d}_{j}^{\mathrm{c}} \boldsymbol{n}_{k}^{\mathrm{c}} \boldsymbol{d}_{l}^{\mathrm{c}}+k_{t} \boldsymbol{t}_{i}^{\mathrm{c}} \boldsymbol{d}_{j}^{\mathrm{c}} \boldsymbol{t}_{k}^{\mathrm{c}} \boldsymbol{d}_{l}^{\mathrm{c}}\right)
\end{aligned}
$$

where $V$ is the volume of the RVE packing; $N_{\mathrm{c}}$ is the number of interparticle contacts; $\boldsymbol{d}_{i}^{\mathrm{c}}$ is the branch vector; $\boldsymbol{f}_{i}^{\mathrm{c}}$ is the contact force; $\boldsymbol{n}_{i}^{\mathrm{c}}$ and $\boldsymbol{t}_{i}^{\mathrm{c}}$ are the normal and tangential unit vectors at the contact, respectively; and $k_{\mathrm{n}}$ and $k_{\mathrm{t}}$ are the normal and tangential contact stiffnesses, respectively.

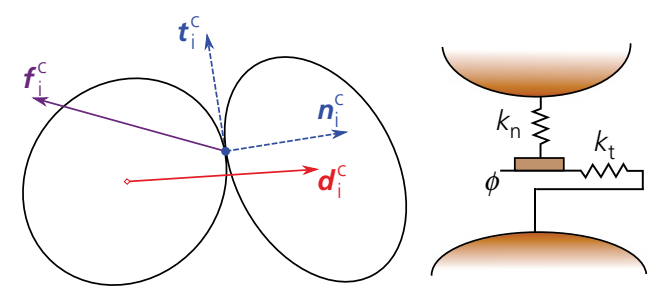

Fig. 1. Illustration of an interparticle contact in DEM
The kinetic and kinematic quantities related to an interparticle contact are illustrated in Fig. 1. In this study, the authors use a linear force-displacement model to calculate the normal force and the Coulomb frictional model for the tangential force with friction angle $\phi$. The contact stiffnesses are determined from two material parameters $E_{\mathrm{c}}$ and $v_{\mathrm{c}}$ by way of: $k_{\mathrm{n}}=2 E_{\mathrm{c}} r_{1} r_{2} /\left(r_{1}+r_{2}\right)$ and $k_{\mathrm{t}}=v_{\mathrm{c}} k_{\mathrm{n}}$, where $r_{1}$ and $r_{2}$ are the radii of the two contacting particles. Details of the solution procedure can be found in Guo \& Zhao (2014).

\section{RESULTS AND DISCUSSION \\ Test set-up}

The domain of the TWHC test is of the same size as that of the experimental counterpart reported in Alsiny et al. (1992), with the cavity radius $r_{\mathrm{c}}=15 \mathrm{~mm}$ and the outer radius $r_{\mathrm{o}}=150 \mathrm{~mm}$. The outer surface of the specimen is subjected to a constant confining pressure $\sigma_{\mathrm{o}}=100 \mathrm{kPa}$. A uniform radial displacement $u_{\mathrm{c}}$ is applied to the inner surface of the specimen to inflate the cavity. Due to the axisymmetry, the cavity problem is often simulated with a quarter of the domain to save computational costs (e.g. Anand \& Gu, 2000; Zervos et al., 2001; François et al., 2014; Desrues et al., 2015). However, due to the grain-scale spatial heterogeneity of the RVEs, non-symmetric bifurcation mode may trigger and break the radial symmetry of the problem. Hence, the authors follow the approach in Papanastasiou \& Vardoulakis (1992) and conduct quarter-domain and full-domain simulations, followed up with a further comparison of their results. The mesh and the boundary conditions for the simulations are illustrated in Fig. 2. For the quarter-domain mesh, 400 serendipity quadrilateral elements are used. The left and the bottom surfaces are subjected to roller boundary conditions. For the full-domain mesh, 1600 serendipity quadrilateral elements are used. Due to the axisymmetry, the hoop displacements of the four symmetric exterior nodes are restrained, as shown in Fig. 2 (Papanastasiou \& Vardoulakis, 1992). Note that a reduced integration technique is used for all the simulations, which requires four Gauss quadrature points for each element (Zhao \& Guo, 2015a).

In this study, the authors use 400 circular particles in the RVE packing with a roughly linear particle radius distribution from 3 to $7 \mathrm{~mm}$. The choice of 400 particles is based on a sensitivity analysis detailed in Guo \& Zhao (2014), and is a compromise between reliable RVE responses and affordable computational cost. Periodic boundary condition is enforced at both directions of the RVE. The particle density is set to $2650 \mathrm{~kg} / \mathrm{m}^{3}$. The two contact stiffness

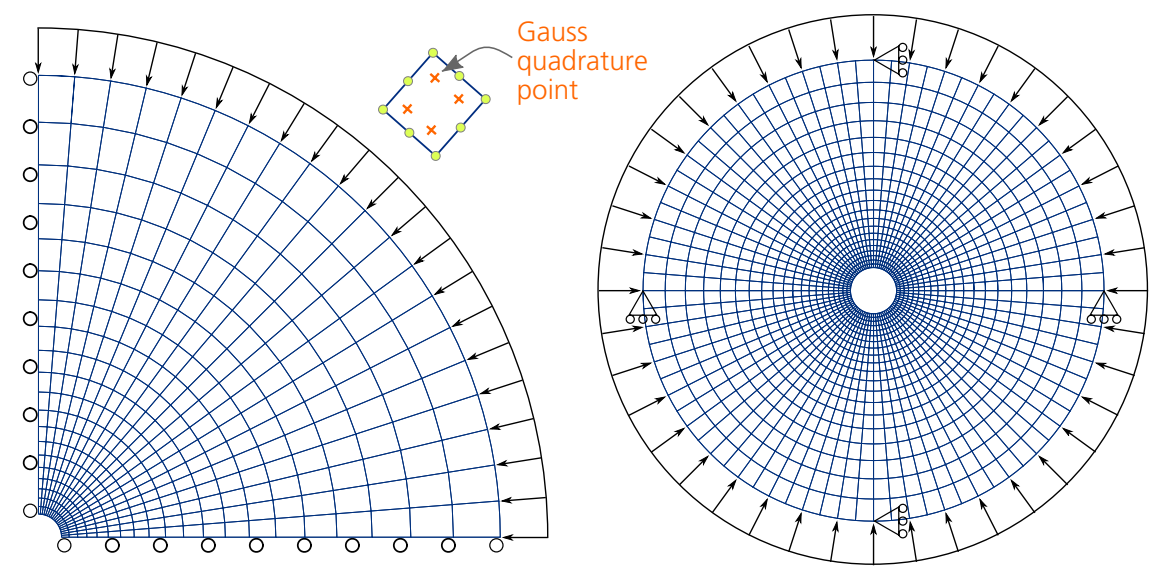

Fig. 2. Mesh and boundary conditions for quarter-domain and the full-domain simulations 
parameters are $E_{\mathrm{c}}=600 \mathrm{MPa}$ and $v_{\mathrm{c}}=0 \cdot 8$. The interparticle friction angle is $\phi=0 \cdot 5$. These material parameters are common in DEM modelling of sands and are consistent with those used in Guo \& Zhao (2014). To reproduce qualitatively the typical responses of dense dry sand, the RVE packing is first isotropically consolidated to a mean effective stress $p_{0}=\sigma_{\mathrm{o}}=100 \mathrm{kPa}$ and to reach a void ratio $e_{0}=0 \cdot 177$. During the sample preparation process, the friction angle is reduced to achieve a relatively dense packing. After the preparation, the RVE packings - with the same initial conditions - are embedded into the Gauss quadrature points of the FEM mesh for the TWHC tests.

\section{Cavity pressure}

The authors first examine the evolution of the cavity pressure $\sigma_{\mathrm{c}}$ against the cavity expansion displacement $u_{\mathrm{c}}$ normalised by the initial cavity radius $r_{\mathrm{c}}$, as plotted in Fig. 3 . It is seen that the results from the simplified quarterdomain and the full-domain simulations are almost identical, despite some small deviations around the peak. Both show an obvious softening post-peak behaviour. From the drained biaxial compression test results on the same RVE packing (Guo \& Zhao, 2014, 2015) shown in Fig. 4, the macroscopic material parameters, such as the peak friction angle $\phi_{\max }^{\prime}$, the peak dilation angle $\psi_{\max }$ and the elastic shear modulus $G$, can be estimated as: $\quad \sin \phi_{\max }^{\prime}=\left(\left(\sigma_{1} / \sigma_{3}\right)_{\max }-1\right) /\left(\left(\sigma_{1} / \sigma_{3}\right)_{\max }+1\right) \approx 0 \cdot 47$, $\sin \psi_{\text {max }}=\left(\left(\mathrm{d} \varepsilon_{1} / \mathrm{d} \varepsilon_{3}\right)_{\text {max }}+1\right) /\left(1-\left(\mathrm{d} \varepsilon_{1} / \mathrm{d} \varepsilon_{3}\right)_{\text {max }}\right) \approx 0 \cdot 26$ (Bolton, 1986) and $G / p_{0} \approx 113$, where $\sigma_{1}\left(\varepsilon_{1}\right)$ and $\sigma_{3}\left(\varepsilon_{3}\right)$ are the major and minor principal stresses (strains), respectively. With these estimations, it is instructive to further compare

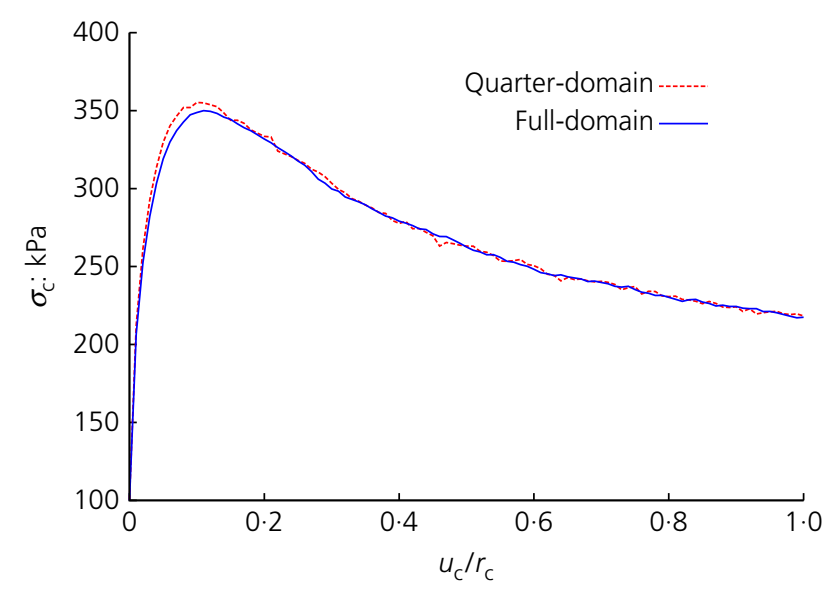

Fig. 3. Cavity pressure against normalised cavity expansion displacement the multiscale predictions with some closed-form solutions for the limit cavity pressure (Carter et al., 1986; Yu \& Houlsby, 1991). According to Yu \& Houlsby (1991), the limit cavity pressure would reach about $15 p_{0}$ (see Appendix) for the cohesionless sand based on an elasto-perfect-plastic constitutive model using the above estimated macroscopic material parameters. However, the current study gives a peak cavity pressure of only $3 \cdot 5 p_{0}$, as seen from Fig. 3 , which is noticeably smaller than the analytical solution. The difference is mainly owing to the softening response of the material, as well as the highly non-uniform deformation field developed in the specimen due to strain localisation (other factors such as boundary condition and assumed two-dimensional simulation may also contribute). These two major factors violate the assumptions adopted in deriving the analytical solution. Indeed, from the experiments conducted by Alsiny et al. (1992) where strain localisation was observed, the cavity pressure can only reach up to a peak value slightly larger than $4 p_{0}$ from different tests - with $p_{0}$ varying from 50 to $200 \mathrm{kPa}$ - followed by a significant decreasing pressure, which are similar to the observations in this study. The complexities of the material response and the deformation field impede an accurate prediction of the limit cavity pressure based on simple assumptions, let alone a reliable prediction of the failure pattern. Therefore, a comprehensive numerical study on the failure mechanism and failure pattern is needed.

\section{Strain localisation}

Shear failure has been observed from both quarter-domain and full-domain simulations of the TWHC tests, where the failure pattern manifests as a series of curvilinear shear bands, as shown in Figs 5(a) and 5(b), in terms of the void ratio contours at two loading stages $\left(u_{\mathrm{c}}=7 \cdot 5\right.$ and $\left.15 \mathrm{~mm}\right)$ (The entire development history of the shear bands can be found in the supplementary material of the paper.). For the quarter-domain simulation (Fig. 5(a)), a primary shear band is found emanating from the middle of the inner surface, propagating upwards along a spiral path, touching the left boundary and getting reflected before finally reaching the exterior surface (see the supplementary animation). The reflective boundary is artificially introduced by the imposed boundary condition that enforces axisymmetry in the quarter-domain modelling. This artificial effect is eliminated in the full-domain simulation (Fig. 5(b)) where four major antisymmetric shear bands have been observed, which are all initiated from the inner surface and develop uninterruptedly towards the exterior surface by following similar spiral patterns. A series of small curvilinear shear bands are also formed and these are intercepted by the primary shear bands. Figure 5(c) compares these two results with those
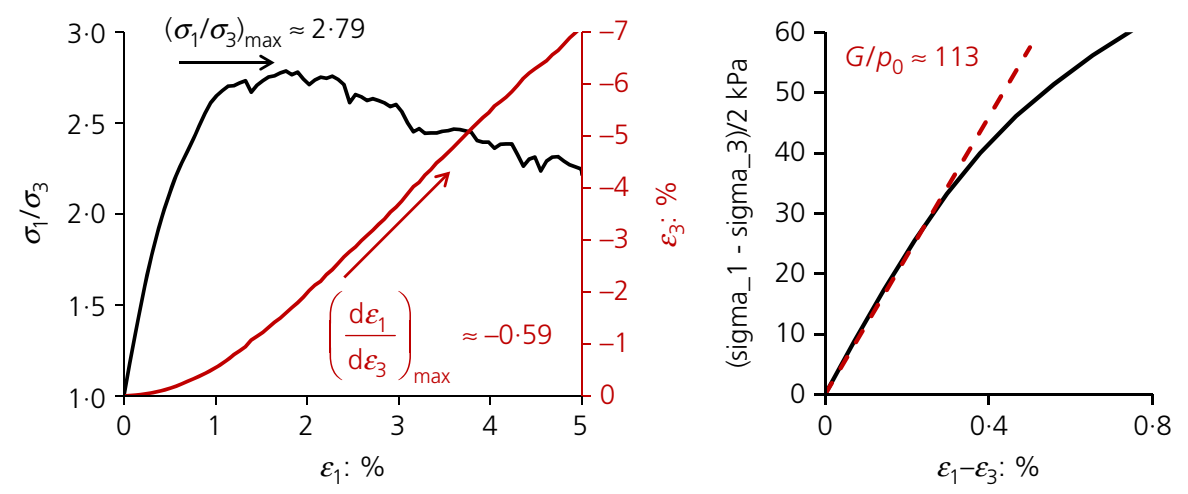

Fig. 4. Stress-strain relations and dilation from the drained biaxial compression test on the RVE 

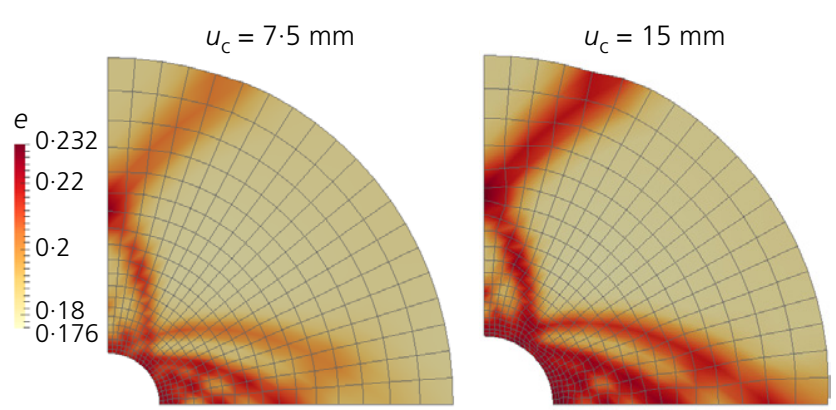

(a)
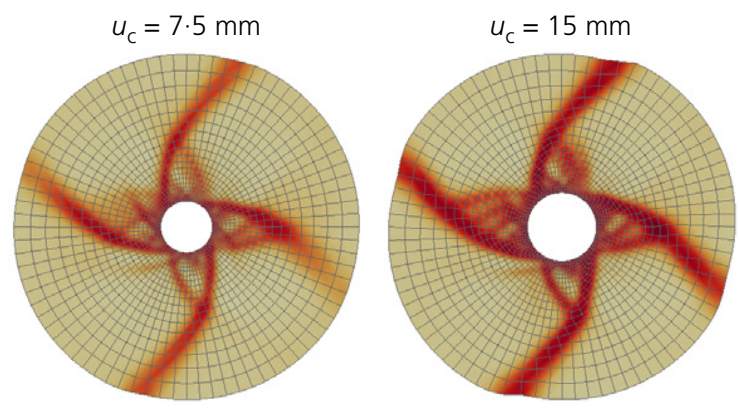

(b)
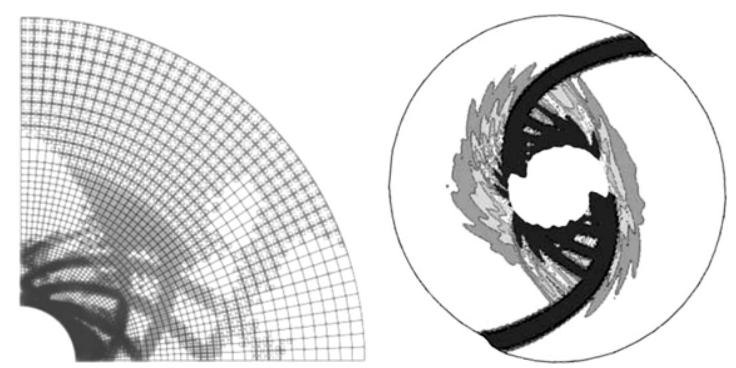

(c)

Fig. 5. Contours of void ratio at $u_{\mathrm{c}}=7 \cdot 5$ and $15 \mathrm{~mm}$ showing strain localisation of (a) the quarter-domain and (b) the fulldomain simulations; (c) comparison with other studies (figures adapted from François et al. (2014) and Crook et al. (2003), respectively)

from Crook et al. (2003) and François et al. (2014), which are obtained by way of macroscopic plasticity models. The authors' quarter-domain and full-domain simulations qualitatively agree well with the results obtained by using plasticity models.

Previous laboratory tests using digital image correlation and X-ray $\mu \mathrm{CT}$ (Rechenmacher, 2006; Hall et al., 2010) revealed that significant particle rotations take place inside the dilative shear band(s). This significant particle rotation is also observed in DEM simulations (Bardet \& Proubet, 1991; Sun et al., 2013a). In the multiscale model, the time history of the motion of each particle in the RVE packings is recorded. This unique aspect of the multiscale model enables us to measure the average particle rotation $\bar{\theta}$ of an RVE packing (averaged over all particles within the packing, positive for anti-clockwise rotation). Figure 6 shows the contours from the two tests at $u_{\mathrm{c}}=15 \mathrm{~mm}$. Clearly, particle rotations are concentrated at the localised dilative zones.

\section{Microstructural analyses}

The multiscale framework offers a convenient way for cross-scale analyses. Under the hood of macroscopic continuum modelling, the microstructure of the material points

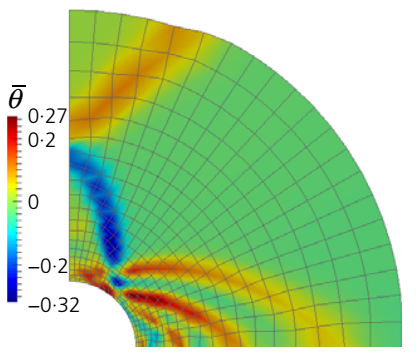

(a)

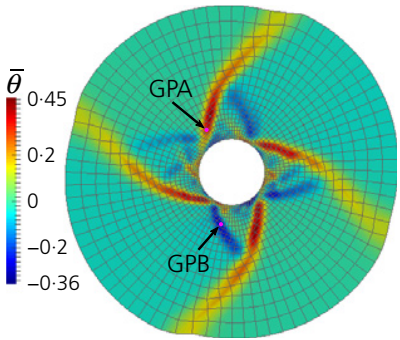

(b)
Fig. 6. Contours of average particle rotation at $u_{\mathrm{c}}=15 \mathrm{~mm}$ of (a) the quarter-domain and (b) the full-domain simulations; GPA and GPB marked in (b) will be used for microstructural analyses

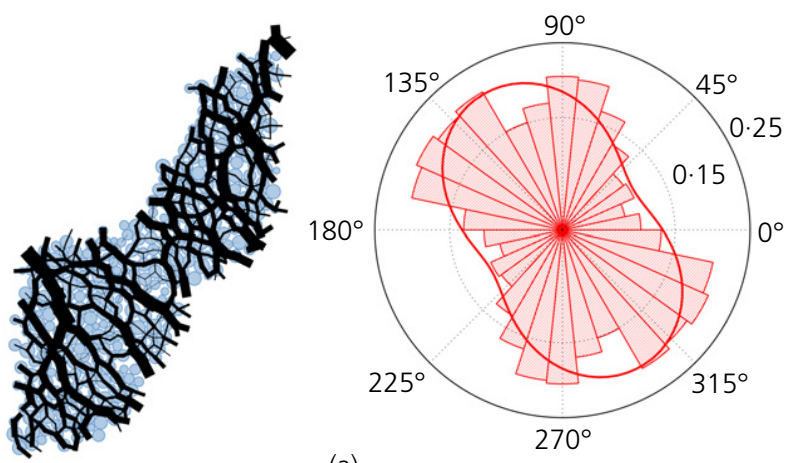

(a)

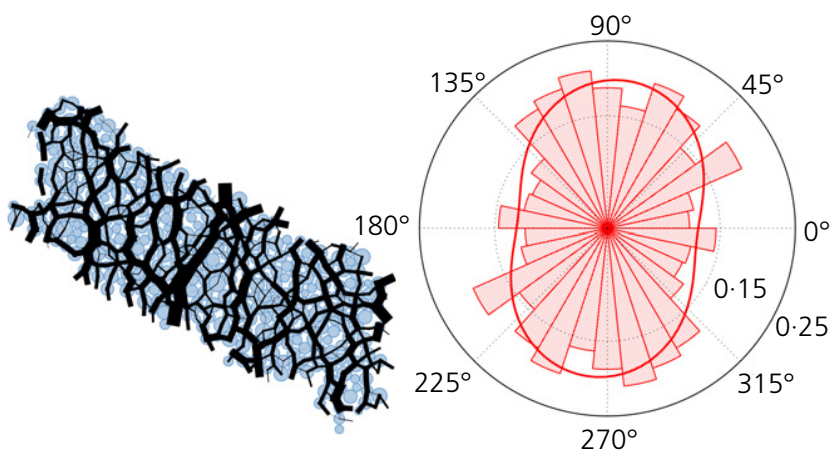

(b)

Fig. 7. Microstructures of local material points in terms of force chains and contact-normal distributions for (a) GPA and (b) GPB at $u_{\mathrm{c}}=15 \mathrm{~mm}$

can be characterised from the embedded RVE packings. Two such packings from different Gauss points of the full-domain simulation are chosen for demonstration. The two points - GPA and GPB - are located inside one of the primary (with positive $\bar{\theta}$ ) and one of the secondary (with negative $\vec{\theta}$ ) shear bands (Fig. 6(b)), respectively. Their microstructures after the cavity expansion at $u_{\mathrm{c}}=15 \mathrm{~mm}$ (The entire evolution history of the force chains at these two points can be found from the animated video in the supplementary material of the paper.) are characterised by the force chains and the contact-normal distributions (or the so-called fabric tensor, Satake, 1982; Guo \& Zhao, 2013; Zhao \& Guo, 2013; Kuhn et al., 2015), as shown in Fig. 7. First, it is seen that the RVE packings at both locations are severely stretched and rotated. The overall packing rotation of GPA is found to be anticlockwise, whereas that of GPB is clockwise, which are consistent with $\bar{\theta}$ at these two points. Second, distinct strong force chains (whose linewidth is proportional to the normal contact force) are observed 
penetrating both packings, indicating that these two packings possess highly anisotropic structures. The major principal direction of the contacts can be seen from the rose diagram of the contact-normal distributions. At both locations, the major principal contact direction of the deformed RVE appears to be consistent with that of the strong force chains.

\section{CONCLUSIONS}

The authors have presented multiscale simulations of the TWHC tests in dry sand. Without the need of any macroscopic phenomenological model, the multiscale approach is capable of reproducing complex shear failure patterns manifested by a series of curvilinear shear bands observed in the TWHC experiments. The cavity pressure limit predicted by the multiscale model is noticeably smaller than its analytical counterpart, which is derived from a simple elasto-perfect-plastic model in the absence of shear banding. The multiscale approach is particularly suitable for macroscale and microscale bridging. The microstructures of local material points and their evolutions under shearing can be conveniently examined. The simulation results indicate that significant particle rotation may develop inside shear bands and lead to highly anisotropic microstructures. One limitation of the study arises from the use of circular particles in the DEM model. For quantitative modelling of sand behaviours, realistic particle shape needs to be considered and this will be a future pursuit of the study.

\section{APPENDIX}

The simplified form of the cylindrical cavity pressure limit of a cohesionless elasto-perfect-plastic soil can be derived by assuming zero elastic strain within the plastic zone $(\mathrm{Yu} \&$ Houlsby, 1991)

$$
\frac{(\alpha+1) \sigma_{\mathrm{c}}}{2 \alpha p_{0}}=\left[\frac{1}{1-(1-\delta)^{(\beta+1) / \beta}}\right]^{1 / \gamma}
$$

where the four material strength coefficients are defined as: $\quad \alpha=\left(1+\sin \phi_{\max }^{\prime}\right) /\left(1-\sin \phi_{\max }^{\prime}\right), \quad \beta=\left(1+\sin \psi_{\max }\right) /$ $\left(1-\sin \psi_{\max }\right), \quad \gamma=\alpha(\beta+1) /[(\alpha-1) \beta] \quad$ and $\delta=(\alpha-1) p_{0} /$ $[2(\alpha+1) G]$. By substituting the estimated macroscopic strength parameters, these four coefficients are obtained as $\alpha \approx 2 \cdot 77, \beta \approx 1 \cdot 7, \gamma \approx 2 \cdot 48$ and $\delta \approx 0 \cdot 002$. Then the cavity pressure limit can be calculated from equation (3) as $\sigma_{\mathrm{c}} \approx 15 p_{0}$. This value is consistent with the range given in Figs 7-9 in Yu \& Houlsby (1991).

\section{ACKNOWLEDGEMENTS}

This study was financially supported by a GRF grant (project number 623211) from Research Grants Council of Hong Kong and a Theme-based Research Project (number T22-603/15-N) from University Grants Committee of Hong Kong. W.C.S. also acknowledges the financial support from the Earth Materials and Processes program at the US Army Research Office under grant contracts W911NF-14-1-0658 and 67433-EV-YIP.

\section{REFERENCES}

Alsiny, A., Vardoulakis, I. \& Drescher, A. (1992). Deformation localization in cavity inflation experiments on dry sand. Géotechnique 42, No. 3, 395-410, http://dx.doi.org/10.1680/ geot.1992.42.3.395.

Anand, L. \& Gu, C. (2000). Granular materials: constitutive equations and strain localization. J. Mech. Phys. Solids 48, No. 8, 1701-1733.
Andrade, J. E., Avila, C. F., Hall, S. A., Lenoir, N. \& Viggiani, G. (2011). Multiscale modeling and characterization of granular matter: from grain kinematics to continuum mechanics. J. Mech. Phys. Solids 59, No. 2, 237-250.

Bardet, J. P. \& Proubet, J. (1991). A numerical investigation of the structure of persistent shear bands in granular media. Géotechnique 41, No. 4, 599-613, http://dx.doi.org/10.1680/geot. 1991.41.4.599.

Bolton, M. D. (1986). The strength and dilatancy of sands. Géotechnique 36, No. 1, 65-78, http://dx.doi.org/10.1680/geot. 1986.36.1.65

Carter, J. P., Booker, J. R. \& Yeung, S. K. (1986). Cavity expansion in cohesive frictional soils. Géotechnique 36, No. 3, 349-358, http://dx.doi.org/10.1680/geot.1986.36.3.349.

Crook, T., Willson, S., Yu, J. G. \& Owen, R. (2003). Computational modelling of the localized deformation associated with borehole breakout in quasi-brittle materials. J. Pet. Sci. Engng 38, No. 3-4, 177-186.

Desrues, J., Nguyen, T. K., Combe, G. \& Caillerie, D. (2015). FEM $\times$ DEM multi-scale analysis of boundary value problems involving strain localization. In Bifurcation and degradation of geomaterials in the new millennium (eds K. T. Chau \& J. Zhao), pp. 259-265. Springer Series in Geomechanics and Geoengineering. Hong Kong: Springer International Publishing.

François, B., Labiouse, V., Dizier, A., Marinelli, F., Charlier, R. \& Collin, F. (2014). Hollow cylinder tests on Boom clay: modelling of strain localization in the anisotropic excavation damaged zone. Rock Mech. Rock Engng 47, No. 1, 71-86.

Guo, N. (2014). Multiscale characterization of the shear behavior of granular media. PhD thesis, The Hong Kong University of Science and Technology, Hong Kong.

Guo, N. \& Zhao, J. (2013). The signature of shear-induced anisotropy in granular media. Comput. Geotech. 47, 1-15.

Guo, N. \& Zhao, J. (2014). A coupled FEM/DEM approach for hierarchical multiscale modelling of granular media. Int. J. Numer. Methods Engng 99, No. 11, 789-818.

Guo, N. \& Zhao, J. (2015). Multiscale insights into classical geomechanics problems. Int. J. Numer. Anal. Methods Geomech., http://dx.doi.org/10.1002/nag.2406.

Hall, S. A., Bornert, M., Desrues, J., Pannier, Y., Lenoir, N., Viggiani, G. \& Bésuelle, P. (2010). Discrete and continuum analysis of localised deformation in sand using X-ray $\mu \mathrm{CT}$ and volumetric digital image correlation. Géotechnique 60, No. 5, 315-322, http://dx.doi.org/10.1680/geot.2010.60. 5.315.

Kaneko, K., Terada, K., Kyoya, T. \& Kishino, Y. (2003). Globallocal analysis of granular media in quasi-static equilibrium. Int. J. Solids Struct. 40, No. 15, 4043-4069.

Kuhn, M. R., Sun, W. \& Wang, Q. (2015). Stress-induced anisotropy in granular materials: fabric, stiffness, and permeability. Acta Geotech. 10, No. 4, 399-419.

Liu, Y., Sun, W. \& Fish, J. (2015a). Determining material parameters for critical state plasticity models based on multilevel extended digital database. J. Appl. Mech. ASME 83, No. 1, 011003.

Liu, Y., Sun, W., Yuan, Z. \& Fish, J. (2015b). A nonlocal multiscale discrete-continuum model for predicting mechanical behavior of granular materials. Int. J. Numer. Methods Engng, http://dx.doi. org/10.1002/nme.5139.

Mántaras, F. M. \& Schnaid, F. (2002). Cylindrical cavity expansion in dilatant cohesive-frictional materials. Géotechnique 52, No. 5, 337-348, http://dx.doi.org/10.1680/geot.2002.52.5.337.

Miehe, C. \& Dettmar, J. (2004). A framework for micro-macro transitions in periodic particle aggregates of granular materials. Comput. Methods Appl. Mech. Engng 193, No. 3-5, $225-256$.

Nguyen, T. K., Combe, G., Caillerie, D. \& Desrues, J. (2014). FEM $\times$ DEM modelling of cohesive granular materials: numerical homogenisation and multi-scale simulations. Acta Geophys. 62, No. 5, 1109-1126.

O'Sullivan, C., Wadee, M. A., Hanley, K. J. \& Barreto, D. (2013). Use of DEM and elastic stability analysis to explain the influence of the intermediate principal stress on shear strength. Géotechnique 63, No. 15, 1298-1309, http://dx.doi.org/10.1680/ geot.12.P.153. 
Papanastasiou, P. C. \& Vardoulakis, I. G. (1989). Bifurcation analysis of deep boreholes: II. Scale effect. Int. J. Numer. Anal. Methods Geomech. 13, No. 2, 183-198.

Papanastasiou, P. C. \& Vardoulakis, I. G. (1992). Numerical treatment of progressive localization in relation to borehole stability. Int. J. Numer. Anal. Methods Geomech. 16, No. 6, 389-424.

Rechenmacher, A. L. (2006). Grain-scale processes governing shear band initiation and evolution in sands. J. Mech. Phys. Solids 54, No. 1, 22-45.

Salgado, R., Mitchell, J. K. \& Jamiolkowski, M. (1997). Cavity expansion and penetration resistance in sand. $J$. Geotech. Geoenviron. Engng 123, No. 4, 344-354.

Satake, M. (1982). Fabric tensor in granular materials. In Deformation and failure of granular materials (eds P. A. Vermeer \& H. J. Luger), pp. 63-68. Rotterdam, the Netherlands: Balkema.

Sun, W. (2013). A unified method to predict diffuse and localized instabilities in sands. Geomech. Geoengng 8, No. 2, $65-75$.

Sun, W. (2015). A stabilized finite element formulation for monolithic thermo-hydro-mechanical simulations at finite strain. Int. J. Numer. Methods Engng 103, No. 11, 798-839.

Sun, W., Kuhn, M. R. \& Rudnicki, J. W. (2013a). A multiscale DEM-LBM analysis on permeability evolutions inside a dilatant shear band. Acta Geotech. 8, No. 5, 465-480.
Tordesillas, A. (2007). Force chain buckling, unjamming transitions and shear banding in dense granular assemblies. Philos. Mag. 87, No. 32, 4987-5016.

Vardoulakis, I. G. \& Papanastasiou, P. C. (1988). Bifurcation analysis of deep boreholes: I. Surface instabilities. Int. J. Numer. Anal. Methods Geomech. 12, No. 4, 379-399.

$\mathrm{Yu}$, H. S. (2000). Cavity expansion methods in geomechanics. Dordrecht, The Netherlands: Springer.

$\mathrm{Yu}$, H. S. \& Houlsby, G. T. (1991). Finite cavity expansion in dilatant soils: loading analysis. Géotechnique 41, No. 2, 173-183, http://dx.doi.org/10.1680/geot.1991.41.2.173.

Zervos, A., Papanastasiou, P. \& Vardoulakis, I. (2001). Modelling of localisation and scale effect in thick-walled cylinders with gradient elastoplasticity. Int. J. Solids Struct. 38, No. 30-31, 5081-5095.

Zhao, J. \& Guo, N. (2013). Unique critical state characteristics in granular media considering fabric anisotropy. Géotechnique 63, No. 8, 695-704, http://dx.doi.org/10.1680/geot.12.P.040.

Zhao, J. \& Guo, N. (2015a). The interplay between anisotropy and strain localisation in granular soils: a multiscale insight. Géotechnique 65, No. 8, 642-656, http://dx.doi.org/10.1680/geot. 14.P.184.

Zhao, J. D. \& Guo, N. (2015b). Bridging the micro and macro for granular media: a computational multi-scale paradigm. In Geomechanics from Micro to Macro (eds K. Soga, K. Kumar, G. Biscontin \& M. Kuo (eds)), pp. 747-752. London, UK: Taylor \& Francis Group. 